\title{
Nucleus Accumbens CREB Activity is Necessary for Nicotine Conditioned Place Preference
}

\author{
Darlene H Brunzell ${ }^{1,2}$, Yann S Mineur', Rachael L Neve ${ }^{3}$ and Marina R Picciotto*,' \\ 'Department of Psychiatry, Yale University School of Medicine, New Haven, CT, USA; ²Department of Pharmacology and Toxicology, Virginia \\ Commonwealth University, Richmond, VA, USA; ${ }^{3}$ Department of Psychiatry, McLean Hospital, Harvard Medical School, Belmont, MA, USA
}

\begin{abstract}
The ability of nicotine to alter firing of dopamine neurons is the first step leading to nicotine reward, but activation of intracellular signaling pathways downstream of nicotinic acetylcholine receptors is likely to be critical for longer-term consequences of nicotine exposure, including conditioned reward. The transcription factor cyclic AMP-response element binding protein (CREB) is important for new gene transcription and in its phosphorylated form (PCREB) promotes long-term changes in synaptic strength. Previous studies have implicated nucleus accumbens (NAc) CREB activity in the modulation of cocaine and morphine reward, and have shown that nicotine conditioned place preference (CPP) is associated with NAc CREB activation. It is not clear whether CPP elicits phosphorylation of CREB or if elevations in PCREB support nicotine CPP. In the current study, we investigated levels of CREB and pCREB during Pavlovian conditioning with nicotine in a novel context in the absence of chamber choice. Nicotine context conditioning resulted in elevated pCREB levels in the NAc shell but not the NAc core of mice following placement in the nicotine-paired chamber in the absence of nicotine. To test if CREB activity in the NAc shell contributes to cue-induced responses that may precipitate nicotine-seeking, we used viral-mediated gene transfer of a dominant-negative CREB construct in the NAc shell of C57BL/6) mice and found that disruption of CREB activation before training blocked nicotine place preference across a range of doses. Taken together, these studies identify the NAc shell as a brain region where CREB activity is essential for nicotine CPP.

Neuropsychopharmacology (2009) 34, 1993-200 I; doi: I0.1038/npp.2009. I I; published online II February 2009
\end{abstract}

Keywords: nicotinic; dopamine; addiction; context; place conditioning; learning

\section{INTRODUCTION}

Despite declines over the past 25 years, more than a fifth of the US population continues to smoke, and an increasing number of tobacco users are intermittent smokers in social settings ('chippers'). It is likely that environmental cues contribute to social and habitual tobacco use. Nicotine, the primary addictive substance in tobacco smoke, stimulates neuronal activity in the ventral tegmental area and downstream dopamine (DA) projection regions that support addictive behavior (Due et al, 2002; Brody et al, 2004). Mesolimbic DA release is believed to underlie the primary reinforcing effects of drugs of abuse including nicotine (Roberts et al, 1980; Di Chiara and Imperato, 1988; Corrigall et al, 1992; Picciotto et al, 1998). In particular, DA release in the nucleus accumbens (NAc) shell is thought to regulate motivation for drug and natural rewards (Di Chiara et al, 2004). Rodent and nonhuman primate studies show that after repeated pairings of a primary reward with a

\footnotetext{
*Correspondence: Dr MR Picciotto, Department of Psychiatry, Yale University School of Medicine, CMHC 304, 34 Park Street, New Haven, CT 6508, USA, Tel: + I 203737 204 I, Fax: + I 203737 2043, E-mail: marina.picciotto@yale.edu

Received 22 September 2008; revised 12 December 2008; accepted 31 December 2008
}

conditioned stimulus (CS) cue, the CS functionally replaces the primary reinforcer in regulation of DA neuron firing (Schultz et al, 1997; Day et al, 2007). In addicted individuals, the rewarding properties of cues associated with a primary reinforcer such as nicotine are thought to have a more predominant function than primary reinforcement in compulsive drug use (Robinson and Berridge, 1993). Associative learning between drug and conditioned rewards involves the amygdala, cingulate cortex, hippocampus and NAc core and shell (Robbins and Everitt, 2002; Robinson and Berridge, 2003), DA projection areas activated by nicotine and cigarette-associated cues in smokers (Due et al, 2002; Brody et al, 2004) and the striatum, including the NAc, appears to regulate cueinduced craving (David et al, 2007; Franklin et al, 2007; McClernon et al, 2008; Volkow et al, 2008). Rodent studies show that a nicotine-associated context stimulates immediate early gene activation in several mesolimblic DA projection areas (Schroeder et al, 2001; Schiltz et al, 2005), suggesting that neuroplasticity associated with nicotine addiction occurs at a molecular level in the DA system.

A candidate gene regulated in the NAc by in vivo nicotine effects on DA is the transcription factor cyclic AMP-response element binding protein (CREB) (Pandey et al, 2001; Brunzell et al, 2003; Pluzarev and Pandey, 2004; Walters et al, 2005). D1- and D2-type DA receptors are 
distinguished according to their respective stimulatory and inhibitory effects on adenylyl cyclase and subsequent activation of CREB by phosphorylation of the protein at Ser-133 (Gomperts et al, 2002). In a nicotine place conditioning paradigm where animals are given a choice between two chambers previously paired with either nicotine or saline, mice show elevated expression of the phosphorylated form of CREB (pCREB) in the NAc that corresponds with increases in preference for the nicotinepaired chamber (Walters et al, 2005). This nicotine conditioned place preference (CPP) is also associated with elevated levels of pCREB in the hippocampus (Walters et al, 2005), a brain area that is important for context conditioning (Kim and Fanselow, 1992; Phillips and LeDoux, 1992) and sensitive to nicotine exposure (Levin et al, 2006; Kenney and Gould, 2008). It is not clear, however, whether phosphorylation of CREB precipitates nicotine chamber choice during CPP or whether the animals' nicotine-seeking behavior results in phosphorylation of CREB. Changes in signaling that precede CPP may be involved in behavioral responses to cue reward. It is interesting that chronic nicotine exposure results in decreases in PCREB in the NAc (Brunzell et al, 2003) and that the rewarding effects of elicit drugs of abuse, such as morphine and cocaine, are enhanced by disruption of CREB activation in the NAc shell (Carlezon et al, 1998; Barrot et al, 2002). Constitutive, global knockout of CREB $\alpha / \delta$ isoforms prevents nicotine CPP (Walters et al, 2005), suggesting that CREB activation is critical for this reward-associated behavior; however the function of $N A c$ CREB activation in nicotine CPP remains to be determined.

To address the function of CREB in nicotine place conditioning, we first tested whether nicotine or a nicotinepaired chamber would regulate total CREB or PCREB levels in the hippocampus and NAc of animals isolated to the training chamber during training or $24 \mathrm{~h}$ following 3 days of nicotine context conditioning in the absence of nicotine. To expand on previous research (Brunzell et al, 2003; Pluzarev and Pandey, 2004; Walters et al, 2005), we subdivided the NAc into core and shell regions. A nicotine-paired chamber increased levels of PCREB in the NAc shell but not the core or the hippocampus. We hypothesized that changes in CREB signaling that occur while the animal is isolated in a nicotine-paired chamber are CS dependent and necessary for nicotine CPP. To test this hypothesis, we used a herpes simplex viral vector to express a dominantnegative form of CREB with a mutation at the Ser-133 phosphorylation site (mCREB) (Carlezon et al, 1998) or control vector (LacZ) into the NAc shell. Mice showed normal nicotine CPP when infused with LacZ control vector, but shell infusions of mCREB blocked nicotine CPP at a range of doses. Together these data suggest that context-associated changes in CREB activation in the NAC shell are necessary for nicotine CPP.

\section{MATERIALS AND METHODS}

\section{Animals}

Male C57BL/6J (8- to 12-week old) mice were purchased from Jackson Laboratories or bred in our laboratory. Mice were maintained in a temperature-controlled vivarium $\left(21 \pm 2^{\circ} \mathrm{C}\right)$ under a $12: 12 \mathrm{~h}$ light-dark cycle and housed four per cage. Food and fluid were available ad libitum. Mice were habituated to handling for at least 3 days before behavioral testing or biochemical studies. All studies were approved by the Yale University Animal Care and Use Committee and followed the NIH Guide for the Care and Use of Laboratory Animals.

\section{Drug Dosing and Administration}

Mice undergoing place conditioning received i.p. injections of nicotine in $0.9 \%$ saline $(0.04,0.065,0.09,0.175,1.75 \mathrm{mg} /$ $\mathrm{kg}$ (by weight of freebase)) or vehicle immediately before training.

\section{CREB and pCREB Induction Studies During Pavlovian Context Conditioning}

For studies of CREB signaling under Pavlovian conditions, separate groups of mice received nicotine (NIC; $0.09 \mathrm{mg} / \mathrm{kg}$, i.p.) or saline injection (SAL) once daily for 3 days immediately before placement in a $(48 \times 22 \times 18 \mathrm{~cm})$ training chamber for $30 \mathrm{~min}$. Brains of all SAL animals and a portion of NIC mice were harvested $15 \mathrm{~min}$ into the third training session. Approximately $22 \mathrm{~h}$ following the third NIC training session, the remaining mice (NIC Chamber) had brains harvested $15 \mathrm{~min}$ following saline injection and placement into the nicotine-paired chamber. A Med Associates locomotor apparatus measured beam breaks to calculate distance traveled during exposure to the training chambers for subgroups of animals in each of the three conditions.

\section{Western Blot Analysis}

Mouse brains were harvested by rapid decapitation, placed in ice-cold PBS, sectioned in a chilled matrix, and placed in fresh, chilled PBS. The dorsal hippocampus was dissected and 16-gauge punches of NAc core and shell were harvested from $1 \mathrm{~mm}$ brain sections and fast-frozen in dry ice. Tissues and blots were processed as described previously (Brunzell et al, 2003). Protein $(10 \mu \mathrm{g})$ was loaded on a polyacrylamide gradient gel (4-20\%) and transferred to nitrocellulose for immunodetection using polyclonal antisera (Cell Signaling, USA) to CREB $(1: 1000)$ and PCREB (Ser-133; 1:500). Blots were washed and then incubated in $1: 1000$ peroxidase-labeled anti-rabbit secondary antiserum (Vector, USA). For NAc core and shell, ECL detection reagents were applied for $1 \mathrm{~min}$, blots were exposed to XRay film and developed. Equal loading was assured by Ponceau staining, and blots were subsequently incubated in 1:10000 GAPDH monoclonal antiserum (Upstate Biotechnology) and 1:5000 anti-mouse secondary antiserum as a loading control. In the hippocampus, monoclonal pCREB $(1: 500$ in mouse) and CREB (1:1000 in rabbit) (Cell Signaling) antisera were incubated on the same blot. Fluorescence-tagged anti-mouse and -rabbit secondary antibodies of different wavelengths were used (Licor) and were scanned using the Odyssey scanning system and software. 


\section{Unbiased Place Conditioning}

Two conditioning chambers with retractable doors were separated by a neutral chamber. The walls and the floors of the chambers varied but lighting was equilibrated so that there was no baseline preference for either chamber. Time spent in each chamber was recorded by beam breaks and calculated using Med-PC IV software.

On day 0 , baseline, mice received a vehicle injection, were placed in the neutral chamber, and allowed to explore the apparatus for $15 \mathrm{~min}$. Mice with a chamber bias greater than $75 \%$ were dropped from studies. On days 1-3, mice were placed in one conditioning chamber for $30 \mathrm{~min}$ following vehicle injection in the morning, and in the afternoon were placed in the opposite conditioning chamber following an injection of drug; vehicle-injected control subjects received saline injections before placement in either chamber. Mice were counterbalanced for drug-paired chamber according to treatment dose, genotype, and baseline preference. On day 4 , testing, mice were injected with vehicle, placed in the center compartment, and allowed access to both conditioning chambers for $15 \mathrm{~min}$. Baseline and testing sessions took place at an intermediate time between the AM and PM training sessions. Total time spent in each chamber was measured and changes from baseline preference were calculated.

\section{HSV-Mediated Gene Transfer}

HSV-mCREB or HSV-LacZ was infused into the NAc shell 1 day after measurement of baseline preference and 2 days before the initiation of place conditioning. Mice were anesthetized with a mixture of ketamine $(130 \mathrm{mg} / \mathrm{kg}$, i.p.) and xylazine $(13 \mathrm{mg} / \mathrm{kg})$. Bilateral infusion canulae were $1.5 \mathrm{~mm}$ apart and offset by $0.5 \mathrm{~mm}$ in length to target the NAc shell when inserted unilaterally at a $20^{\circ}$ angle, sparing the overlying cingulate cortex and contralateral structures. Stereotaxic placement of a $10 \mathrm{~mm}$ guide canula (o.d./i.d. $0.6 / 0.45 \mathrm{~mm}$ ) into the NAc shell minimized backflow into overlying structures $(1.5 \mathrm{~mm} \mathrm{~A} / \mathrm{P}, 2.5 \mathrm{~mm} \mathrm{~L}$, and $-4.0 \mathrm{~mm}$ $\mathrm{D} / \mathrm{V}$ with respect to bregma). Anatomical control animals received an optimal dose of nicotine following a more dorsal infusion of HSV-mCREB $(1.5 \mathrm{~mm} \mathrm{~A} / \mathrm{P}, 2.25 \mathrm{~mm} \mathrm{~L}$, and $-3.25 \mathrm{~mm} \mathrm{D} / \mathrm{V}$ from dura) to further insure that behavioral effects of mCREB were due to infusion into the NAc shell and not into an overlying structure. HSV vector $(0.7 \mu \mathrm{l}$ over $14 \mathrm{~min})$ was infused by microinfusion pump. Canulae were kept in place for $5 \mathrm{~min}$ after infusion. Following surgery, mice were housed four per cage. A nonsteroidal anti-inflammatory drug $(5 \mathrm{mg} / \mathrm{kg}$, Rimadyl) was used for preemptive analgesia in some mice; all mice received $64 \mathrm{mg}$ of children's acetaminophen applied to wet chow ad libitum during recovery. Most mice recovered from surgery within $48 \mathrm{~h}$ without signs of discomfort, infection, or bleeding. Following 2 days recovery, mice underwent CPP training on days when HSV-mediated mCREB expression was at its peak (days 2-4 post-infusion), and underwent subsequent testing on day 5 (Carlezon et al, 1998; Barrot et al, 2002). Following behavioral procedures, mice received lethal doses of chloral hydrate, were perfused intracardially with chilled $4 \%$ paraformaldehyde (PFA), and brains were harvested and sectioned for verification of canula placement. A separate group of HSV-LacZ mice brains were harvested 3 days post-infusion for the X-gal enzymatic assay.

\section{X-Gal Immunohistochemistry}

Mice were perfused transcardially with ice-cold PBS followed by $4 \%$ PFA in $0.1 \mathrm{~mol} / 1$ phosphate buffer. Brains were removed, postfixed in $4 \%$ PFA, and subsequently placed in $30 \%$ sucrose in PBS for dehydration. Sections $(40 \mu \mathrm{m})$ were collected on a freezing microtome and stored in cryoprotectant at $-20^{\circ} \mathrm{C}$. For X-gal histochemistry, sections were washed $3 \times$ in PBS, incubated for $4 \mathrm{~h}$ at $37^{\circ} \mathrm{C}$ in $\mathrm{PBS}$ solution containing $3.1 \mathrm{mmol} / \mathrm{l}$ potassium ferrocyanide, $1 \mathrm{mmol} / \mathrm{l} \mathrm{MgCl}, 0.01 \%$ sodium deoxycholate, $0.02 \%$ Nonidet P-40, and $0.2 \mathrm{mg} / \mathrm{ml} \mathrm{X}$-gal. $\beta$-Galactosidase enzymatic activity was terminated with $5 \mathrm{~min}$ washes in PBS. Sections were mounted, stained with neutral red, dehydrated with increasing concentrations of ethanol, and coverslipped with Permount.

\section{Statistical Analyses}

For place conditioning, significant effects of treatment and genotype were determined analyzing conditioning chamber as a within-subject measure (drug paired vs saline paired), with between-subjects measures for viral-mediated transfection (HSV-mCREB or HSV-LacZ) and drug (eg NIC/ VEH). Post hoc two-tailed $t$-tests were performed for all statistics except where previous studies predicted a directional finding to justify use of one-tailed $t$-tests. Western blot optical densities were normalized to Vehicle controls to enable comparisons across blots. Planned comparisons were made between vehicle-injected (SAL) and nicotineinjected (NIC) animals and between SAL subjects and animals exposed to a nicotine-paired chamber in the absence of nicotine (NIC Chamber). Just before brain harvest, locomotor activity was assessed between treatment groups (SAL, NIC, and NIC Chamber) using time bin as a repeated measure.

\section{RESULTS}

\section{A Nicotine-Paired Context Leads to Increases in pCREB in the NAc Shell in the Absence of Changes in Locomotor Activity}

Exposure to a nicotine-paired context was sufficient to activate NAc CREB activity in the absence of overt nicotineseeking (Figure 1a). In comparison to saline-injected control animals that received no nicotine exposure, salineinjected animals exposed to a chamber where they had previously received nicotine showed a significant increase in levels of pCREB $(p=0.05)$ in the NAc shell. Slight elevations in NAc shell pCREB levels during nicotine Pavlovian training failed to reach significance $(p>0.1)$, suggesting that the CS properties of the context rather than the primary rewarding properties of the nicotine regulate this effect following 3 days of training. Neither nicotine nor a nicotine-associated context resulted in changes in PCREB levels in the hippocampus or NAc core (Figure $1 \mathrm{~b}$ and $\mathrm{c}$ ) and there was no effect of treatment condition on total 
a
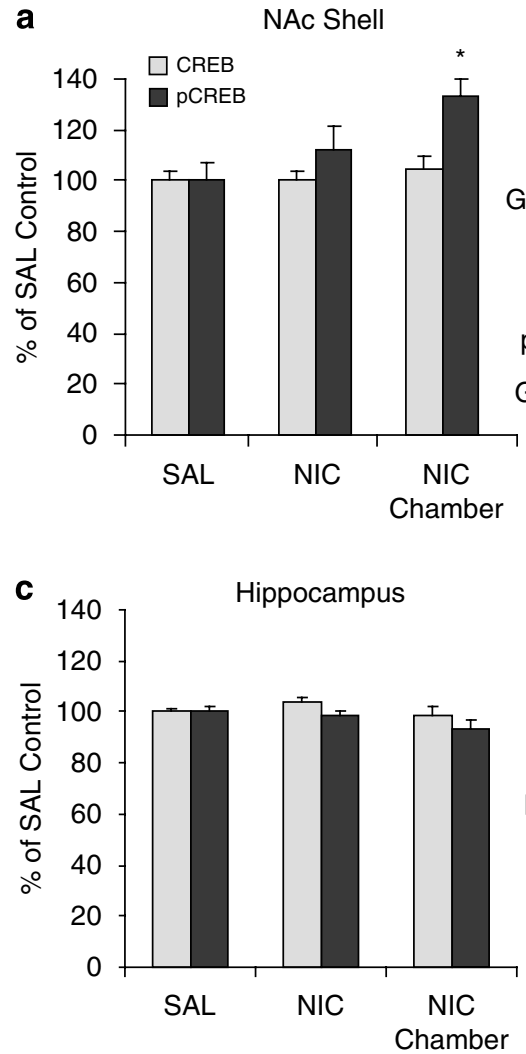

b

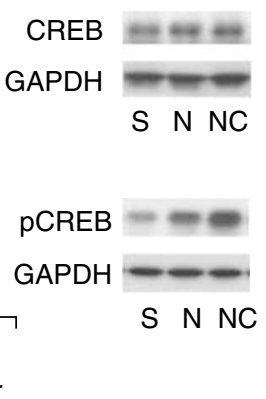

CREB

$S$ N NC

PCREB

$S \mathrm{~N} \mathrm{NC}$
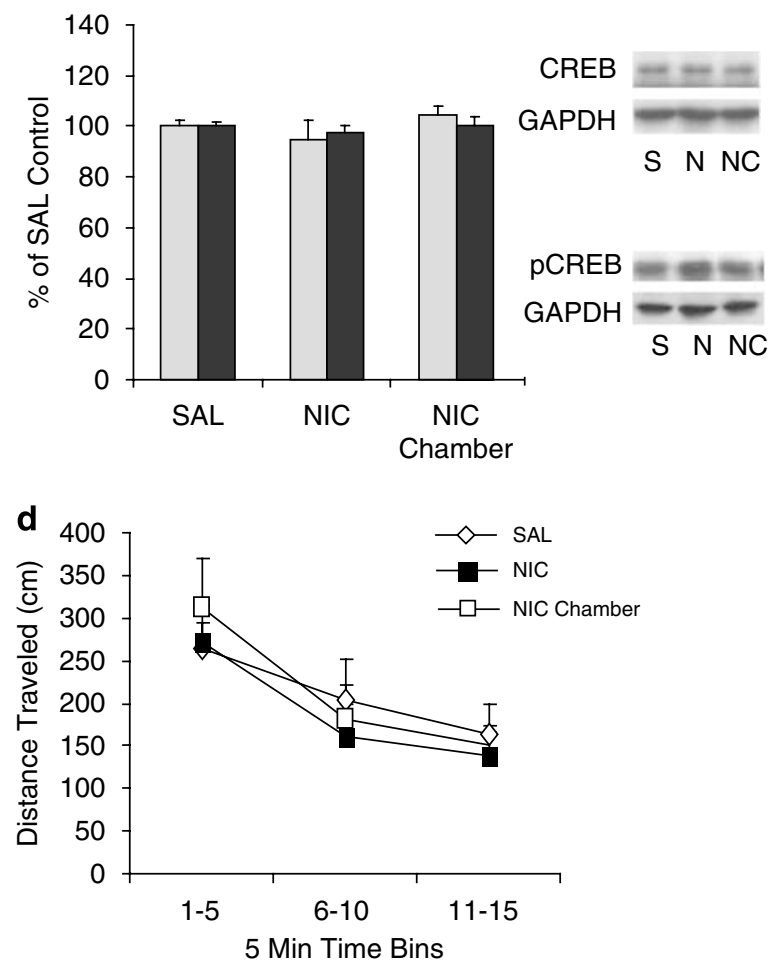

Figure I Nicotine context conditioning increases phosphorylated cyclic AMP-response element binding protein (pCREB) levels in the nucleus accumbens (NAc) shell but not the NAc core or dorsal hippocampus. (a) In comparison to animals injected with saline in the training chamber (SAL, S; $n=12$ ), mice that received nicotine $(0.09 \mathrm{mg} / \mathrm{kg}$, i.p. by weight of freebase) showed no effect of treatment on levels of pCREB during the third day of Pavlovian training (NIC, N; $n=13$ ), but did show NAc shell-specific elevations in PCREB following exposure to the chamber previously paired with nicotine (NIC Chamber, NC; $n=4 ; p<0.05$ ). Total CREB levels in the NAc shell were not regulated by NIC or exposure to the NIC-paired Chamber. (b, $c$ ) There was no effect of $\mathrm{NIC}$, or NIC Chamber exposure on either NAc core or hippocampal levels of CREB or pCREB ( $p$ 's $>0.1$ ). (d) There was no difference in locomotor activity across groups of SAL, NIC, and NIC Chamber mice during the 15 min post-injection immediately before brain harvest $(p>0.1)$. Activity data are presented as distance traveled in $\mathrm{cm}$ across $5 \mathrm{~min}$ time bins. All brains were harvested $15 \mathrm{~min}$ following injection and placement into the chamber. Data from western blotting are presented as percent of SAL control data. Error bars represent SEM; * $p \leqslant 0.05$ compared to SAL animals.

CREB levels in the NAc shell, core, or hippocampus $(p$ 's $>0.1)$.

Figure 1d shows nicotine-associated locomotor activity on the third day of training and during exposure to a nicotine-paired chamber in the absence of nicotine. There was no difference in locomotor activity across experimental conditions on the day of brain harvest $\left(\mathrm{F}_{2,11}=0.448\right.$, $p=0.65$ ), and no interaction of Pavlovian condition with time bin on distance traveled $\left(\mathrm{F}_{2,11}=1.662, p=0.23\right)$. These data further suggest that the nicotine-associated context and not the overt behavior of the animal led to the observed induction of pCREB in the NAc shell.

\section{Unbiased Nicotine CPP is Dose Dependent}

Nicotine CPP in C57BL/6J mice was tightly regulated by dose $\left(\mathrm{F}_{3,39}=2.87, p=0.05\right)$. As previously reported in rats (Le Foll and Goldberg, 2005) and mice (McGeehan and Olive, 2003), C57BL/6J mice showed nicotine CPP at the $0.09 \mathrm{mg} / \mathrm{kg}$, i.p. dose of nicotine as measured by a preference for the drug-paired chamber in comparison to saline controls $(p=0.05$; Figure 2a). The highest dose of nicotine $(0.175 \mathrm{mg} / \mathrm{kg})$ did not result in a preference for the nicotine-paired chamber, presumably due to potential aversive effects of nicotine. Unlike s.c. administration under similar conditions (Walters et al, 2006), however, i.p. nicotine did not result in conditioned place aversion to the nicotine-paired chamber even at a dose of nicotine one $\log$ higher than the highest dose $(1.75 \mathrm{mg} / \mathrm{kg}$, i.p.; data not shown), perhaps due to faster kinetics of the i.p. route of administration.

\section{Decreases in CREB Activity in the NAc Shell Oppose Nicotine Reward Learning}

We used a modified herpes simplex viral vector (HSV) to express mCREB, a dominant-negative form of CREB with a mutation of Ser-133 to alanine, in the NAc shell to test directly whether place-conditioning-associated changes in CREB activity are necessary for nicotine CPP. The serine to alanine mutation in this HSV-mCREB construct reduces cyclic AMP-response element (CRE)-mediated transcription in CRE-LacZ reporter mice (Barrot et al, 2002); hence, mCREB expression disrupts NAc CREB activity and will oppose increases in NAc CREB activity that may occur during place conditioning and following nicotine $\mathrm{CPP}$ (Supplementary Figure S1). There was a significant interaction of HSV infusion with dose of nicotine $\left(\mathrm{F}_{3,148}=2.836\right.$, 

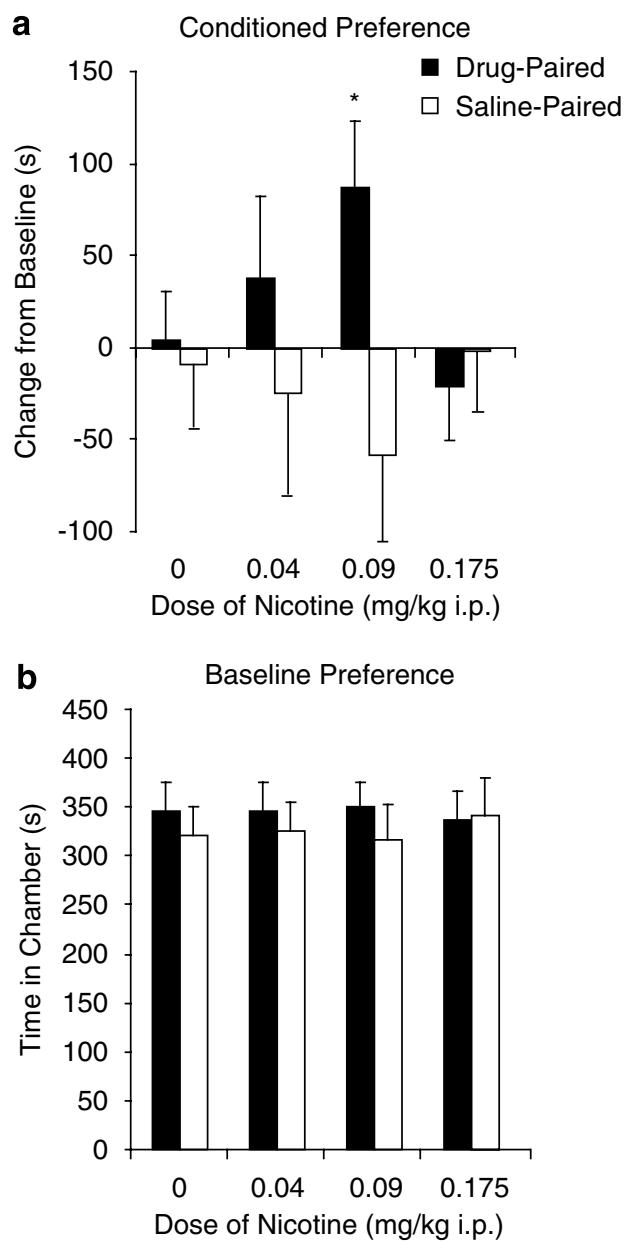

Figure 2 Nonbiased nicotine conditioned place preference (CPP) is tightly regulated by dose. (a) C57BL/6J mice showed a trend for nicotine CPP at the $0.04 \mathrm{mg} / \mathrm{kg}$ dose $(n=12)$, a significant increase in preference for the nicotine-paired chamber at the $0.09 \mathrm{mg} / \mathrm{kg}$ dose $(n=8 ; p=0.05)$, and no preference at the highest dose of i.p. nicotine, $0.175 \mathrm{mg} / \mathrm{kg}(n=8)$ as compared to saline-injected animals $(n=9)$. All nicotine doses are calculated by weight of the freebase. Data are presented in seconds as mean percent change from baseline preference; error bars reflect SEM; * $p<0.05$ compared to vehicle control animals. (b) There was no difference in time spent in the conditioning chamber at baseline indicating that animals showed no overall bias for either chamber before training ( $p$ 's $>0.1$ ), suggesting that change in preference was due to nicotine reward rather than anxiolytic effects of nicotine treatment.

$p=0.04)$ on change in chamber preference. Mice infused with HSV-LacZ control vector, like untreated animals, showed dose-dependent nicotine CPP (Figure 3a), but pretraining NAc shell infusion of HSV-mCREB blocked preference for the nicotine-paired chamber across a range of doses (Figure $3 \mathrm{a}$ and $\mathrm{b}$ ). HSV-mCREB infusion into the NAc shell also resulted in a significant preference for the saline chamber at the optimal dose for nicotine CPP in untreated mice $(0.09 \mathrm{mg} / \mathrm{kg} ; p=0.036)$, which resulted from a shift in time spent in the center compartment and did not reflect a significant decrease in preference for the drug-paired chamber. A subgroup of animals tested for locomotor activity on the day of testing showed no effect of HSV infusion or prior nicotine treatment on locomotor activity (Supplementary Figure S2). Reconstructions of infusion canula placement and an HSV-LacZ X-gal enzymatic activity assay demonstrate that HSV vector expression was local to the site of infusion (Figure 4). Control animals that received infusions $0.75 \mathrm{~mm}$ dorsal to the NAc shell showed similar nicotine CPP to HSV-LaZinfused animals following administration of $0.09 \mathrm{mg} / \mathrm{kg}$, i.p. nicotine, as measured by an increase in preference for the drug-paired chamber over the saline-paired chamber $(p=0.05)$. Preference for the drug-paired chamber was significantly greater in saline-injected controls $\left(t_{54}=1.675\right.$, $p=0.05$ ), suggesting that blockade of nicotine CPP in these experiments chiefly involved inhibition of CREB in the NAc shell and not some overlying structure (Figure 5).

\section{DISCUSSION}

These studies demonstrate that activation of CREB in the NAc shell is critical for nicotine CPP, a form of appetitive contextual conditioning. We further show that a nicotinepaired context increases levels of pCREB in the NAc shell in the absence of overt nicotine-seeking or locomotor activation, suggesting that exposure to an environment associated with nicotine can result in activation of the transcription factor CREB. Context-associated elevations of pCREB were specific to the NAc shell and not observed in the NAc core or the hippocampus using these parameters. Under place conditioning training conditions where animals were given a choice of chamber, disruption of CREB activation in the NAc shell blocked nicotine CPP, suggesting that induction of pCREB by exposure to contextual cues may support nicotine reward learning.

These data expand on previous studies of CREB $\alpha \delta$ knockout mice (Walters et al, 2005) suggesting that regulation of CREB is critical for nicotine CPP. We show that in animals that develop with normal CREB function, inactivation of CREB in the NAc shell in adulthood is sufficient to disrupt nicotine CPP. Previous reports have shown that HSV-mCREB infusion into the NAc shell enhances cocaine and morphine CPP (Carlezon et al, 1998; Walters and Blendy, 2001; Barrot et al, 2002). In contrast, the current study shows that nicotine CPP cannot occur without CREB activity in the NAc shell. This suggests that although various drugs of abuse converge on the NAc in terms of DA release (Di Chiara and Imperato, 1988), the circuit-level adaptations that underlie nicotine reward differ from those of other drugs of abuse (Nestler, 2005). Interestingly, chronic nicotine results in decreases in pCREB in the NAc (Brunzell et al, 2003), and thus may contribute to the ability of cigarette smoking to promote the transition to use of other drugs of abuse (Lai et al, 2000). Other studies, however, show that elevated PCREB is associated with cocaine CPP in the NAc (Miller and Marshall, 2005; Walters et al, 2005) and antisense-induced reductions of NAC CREB in shell or core attenuate the primary reinforcing efficacy of cocaine as well as its ability to act as primer during reinstatement (Choi et al, 2006), perhaps due to more chronic dosing regimens of cocaine self-administration (McClung and Nestler, 2003). Results obtained with CPP do not always translate onto intravenous self-administration data, but unfortunately, reliable nicotine self-administration is difficult to establish in mice. 
a
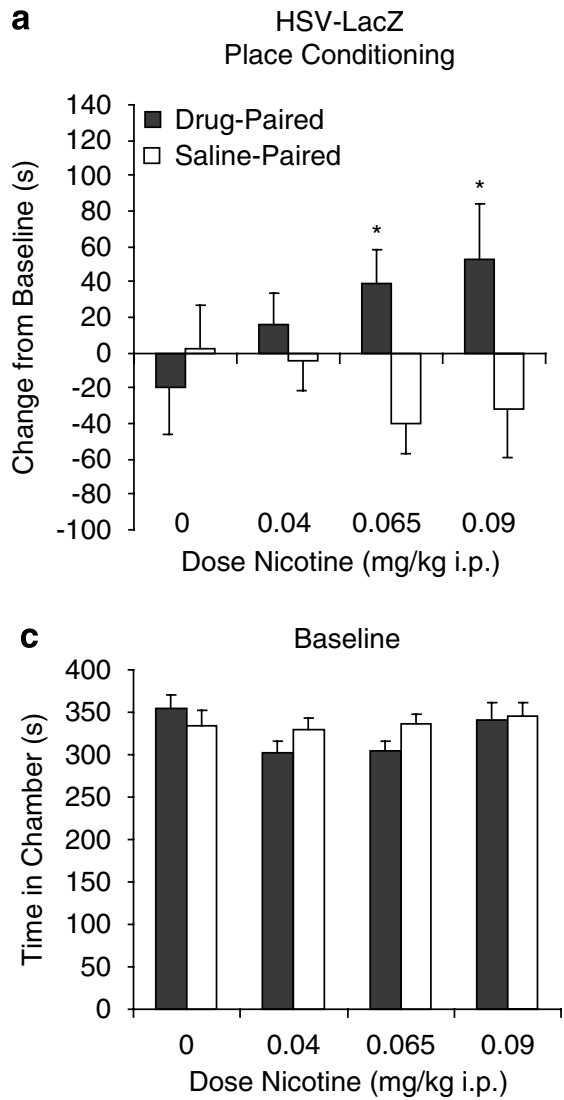

b

HSV-mCREB

Place Conditioning

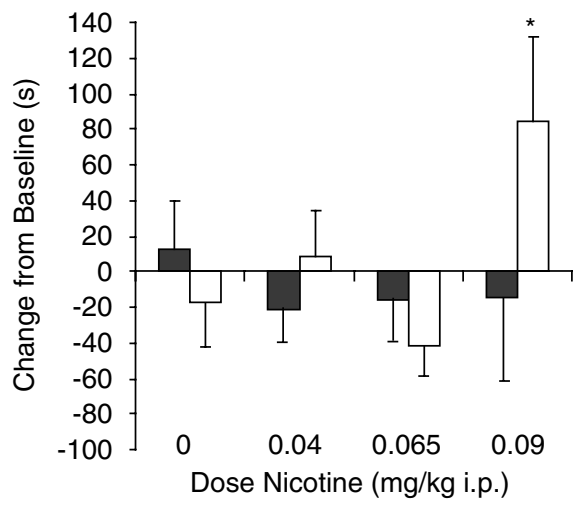

d

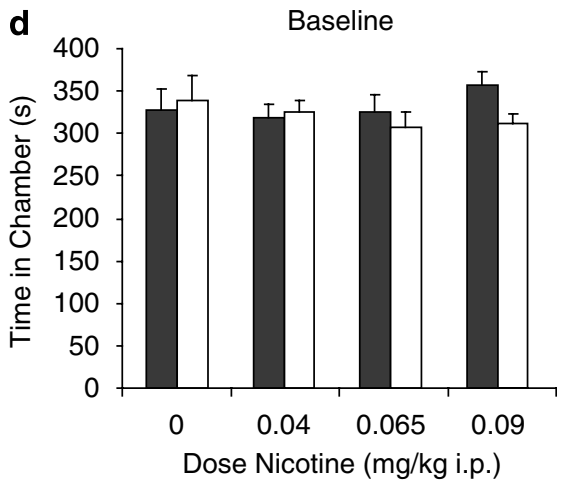

Figure 3 Blockade of cyclic AMP-response element binding protein (CREB) activation in nucleus accumbens (Nac) shell with HSV-mCREB abolishes nicotine conditioned place preference (CPP) across a range of doses. (a) HSV-LacZ-treated mice showed a preference for the nicotine-paired chamber at the $0.065(n=18)$ and $0.09(n=20) \mathrm{mg} / \mathrm{kg}$, i.p. doses of nicotine but not at the $0.04 \mathrm{mg} / \mathrm{kg}$ dose $(n=22)$ as compared to saline-treated controls $(n=18)$. (b) HSV-mCREB infusion into the NAc shell blocked nicotine CPP at all i.p. nicotine doses as demonstrated by the lack of preference for the drug-paired chamber (All p's >0.2; $n=15-23$ ). HSV-mCREB-infused mice showed preference for the saline-paired chamber at the $0.09 \mathrm{mg} / \mathrm{kg}$, i.p. dose of nicotine. (c, d) Mice showed no preference for the drug- or saline-paired chamber at baseline. Data are presented as changes from baseline preference \pm SEM; * $p \leqslant 0.05$ compared to vehicle control mice.

Place conditioning provides a valuable tool for assessing Pavlovian contributions to changes in signaling and consequent reward learning. The present studies expand on earlier data, which did not discriminate between subdivisions of the NAc (Pandey et al, 2001; Brunzell et al, 2003; Walters et al, 2005), to show a dichotomy between the NAc core and shell in nicotine-associated regulation of PCREB. Previous data show that CPP testing, but not a similar regimen of nicotine exposure and withdrawal in the homecage, results in elevations of NAc pCREB (Walters et al, 2005). In the current study mice showed an increase in NAc shell levels of pCREB while isolated to a nicotine-paired chamber $22 \mathrm{~h}$ after the last of 3 daily pairings of nicotine with that chamber. Hence, Pavlovian nicotine place conditioning resulted in elevations of pCREB in the absence of overt nicotine-seeking. Microdialysis studies have shown that elevated DA release, which ought to lead to D1-associated activation of CREB, occurs in the NAc shell but not the core during early acquisition sessions (fewer than seven), indicating that CREB in the NAc shell may contribute to initiation of nicotine use (Spina et al, 2006). These Pavlovian conditioning data support the hypothesis that the CS properties of the context are sufficient to activate CREB in the NAc shell.
At the molecular level, activation of pCREB in the NAc follows a pattern that is similar to that seen for DA neuron activity during stimulus reward learning. Studies in primates and rodents show that after repeated pairing of a rewarding stimulus with a cue, DA neuron firing shifts from responding to the primary rewarding stimulus, becoming dependent instead on the conditioned cue (Schultz et al, 1997; Day et al, 2007). Similarly, elevations of pCREB were observed in the NAc in response to acute nicotine exposure, but not following 4 days of exposure to nicotine in the homecage (Walters et al, 2005) or during Pavlovian training on the third day of nicotine exposure in these studies. Instead, NAc pCREB levels were elevated in response to the nicotine-paired chamber, suggesting that the CS properties of the context and not the rewarding properties of nicotine regulate pCREB in the NAc shell after training that is sufficient to lead to nicotine CPP.

CREB signaling is necessary for neural plasticity associated with learning and addiction (Silva et al, 1998; Carlezon et al, 2005). Genes such as fos and BDNF that are regulated by nicotine and thought to be important for tobacco addiction (Schroeder et al, 2001; Li et al, 2008) are upregulated as a result of CREB overexpression and are inhibited by expression of mCREB in the NAc of transgenic 
a

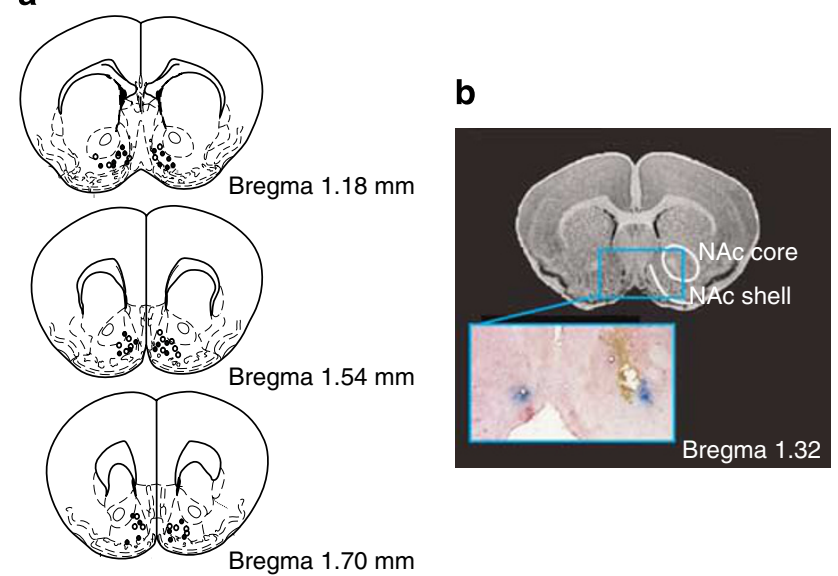

Figure 4 HSV infusions into the nucleus accumbens (NAc) shell. (a) Reconstruction of canulae placement for HSV-mCREB- (closed circles) and HSV-LacZ-infused animals (open circles). (b) A representative mouse NAc section harvested 3 days post-HSV-LacZ infusion. X-gal enzymatic reaction demonstrates that HSV infusions were local to the NAc shell infusion site. The use of guide canulae helped to insure that viral vector that may have traveled up the infusion canulae did not infect overlying tissues. Coronal section of striatum used with permission: Rosen GD, Williams AG, Capra JA, Connolly MT, Cruz B, Lu L et al. (2000). The Mouse Brain Library@ www.mbl.org. Int Mouse Genome Conference 14: 166.

mice (McClung and Nestler, 2003). Disruption of CREB activity could interfere with expression of many genes that have CRE sequences in their promoters. For example, CREB activity is necessary for the regulation of $\mu$-opiate receptors in response to nicotine administration, and this upregulation contributes to nicotine CPP (Walters et al, 2005). Thus, CRE-mediated neuroadaptations that normally occur in response to nicotine may lead to lasting changes in systems that regulate incentive salience and drug reward.

There was no effect of nicotine or a nicotine-paired chamber on regulation of PCREB in the hippocampus in the current study, as was observed using a 4-day regimen of CPP training in previous studies (Walters et al, 2005). One potential reason for this difference may be that animals were not actively seeking the nicotine-paired chamber in the current study, but were rather confined to that chamber by the experimenter. Some evidence suggests that regulation of CREB in the dorsal hippocampus is more critical for spatial navigation than context conditioning (Pittenger et al, 2002). Although CREB was not regulated in the NAc core or hippocampus during exposure to a nicotine-paired chamber on the third day of training, we cannot conclude that CREB in these brain areas is not critical for learning during earlier trials or for consolidation processes related to nicotine CPP (Josselyn and Nguyen, 2005). Human studies show that the dorsal striatum and orbitofrontal cortex may also be involved in cue reward for drugs of abuse (David et al, 2007; Franklin et al, 2007; McClernon et al, 2008; Volkow et al, 2008). Whereas guide canulae prevented expression of mCREB along the canulae tracks, damage to these overlying structures could have impaired cue reward. LacZ control animals showed levels of nicotine CPP similar to untreated controls, however, suggesting that potential unilateral damage during infusion did not affect nicotine CPP.

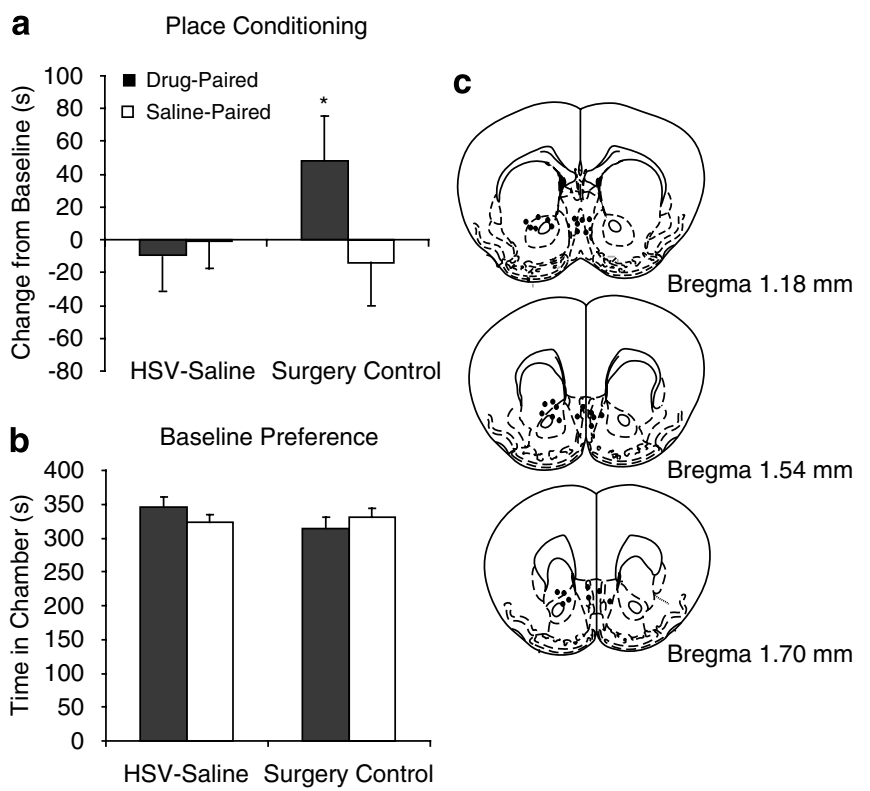

Figure 5 HSV-mCREB infusions into structures above the nucleus accumbens (NAc) shell did not disrupt nicotine conditioned place preference. (a) Surgery control animals $(n=18)$ showed a conditioned place preference to $0.09 \mathrm{mg} / \mathrm{kg}$, i.p. nicotine as measured by a significant increase in preference for the nicotine-paired chamber compared to HSV saline-injected animals $(n=38)$. (b) Mice showed no preference for either chamber at baseline. (c) Reconstructions of canulae placement for HSV$m C R E B$ (closed circles) in animals that received infusions $0.75 \mathrm{~mm}$ dorsal to the NAc shell target structure. Data are presented as mean changes from baseline preference and mean time spent in either chamber at baseline \pm SEM; * $p<0.05$ compared to saline-injected controls.

In summary, these studies show that activation of CREB in the NAc shell is critical for nicotine CPP. The phosphorylation state of CREB may therefore regulate the motivational valence for nicotine or the conditioned rewarding effects of nicotine-associated cues, such as a context associated with nicotine. A nicotine-paired environment can elicit CREB phosphorylation in the NAc shell, suggesting that contextual cues may drive changes in CREB that promotes nicotine reward learning. We conclude from the current study that pharmacological agents that decrease CREB phosphorylation might be novel targets for smoking cessation.

\section{ACKNOWLEDGEMENTS}

We thank J Chang, C Kochevar, Y Pham, B Schneider and C Wein for technical assistance, and William A Carlezon for thoughtful comments on this paper. This work was supported by National Institutes of Health grants AA15632, DA14241, DA10455, a Lieber NARSAD Young Investigator Award to DHB, and DA00436 to MRP. We especially thank Constance and Steven Lieber for their generous support of this work through NARSAD.

\section{DISCLOSURE/CONFLICT OF INTEREST}

The authors have no financial conflicts of interest with any data or original ideas presented in this paper. DH Brunzell 
and RL Neve are supported by the National Institute of Health (NIH) for projects unrelated to the work presented in this paper. YS Mineur is supported by the National Institute of Health and by a Pfizer contract for an unrelated project. In addition to the NIH support of this work and other projects, MR Picciotto has received funding from Pfizer for unrelated research endeavors. YS Mineur and MR Picciotto have a patent on cytisine derivatives as novel treatments for depression.

\section{REFERENCES}

Barrot M, Olivier JD, Perrotti LI, DiLeone RJ, Berton O, Eisch AJ et al (2002). CREB activity in the nucleus accumbens shell controls gating of behavioral responses to emotional stimuli. Proc Natl Acad Sci USA 99: 11435-11440.

Brody AL, Olmstead RE, London ED, Farahi J, Meyer JH, Grossman $\mathrm{P}$ et al (2004). Smoking-induced ventral striatum dopamine release. Am J Psychiatry 161: 1211-1218.

Brunzell DH, Russell DS, Picciotto MR (2003). In vivo nicotine treatment regulates mesocorticolimbic CREB and ERK signaling in C57Bl/6J mice. J Neurochem 84: 1431-1441.

Carlezon Jr WA, Duman RS, Nestler EJ (2005). The many faces of CREB. Trends Neurosci 28: 436-445.

Carlezon Jr WA, Thome J, Olson VG, Lane-Ladd SB, Brodkin ES, Hiroi $\mathrm{N}$ et al (1998). Regulation of cocaine reward by CREB. Science 282: 2272-2275.

Choi KH, Whisler K, Graham DL, Self DW (2006). Antisenseinduced reduction in nucleus accumbens cyclic AMP response element binding protein attenuates cocaine reinforcement. Neuroscience 137: 373-383.

Corrigall WA, Franklin KB, Coen KM, Clarke PB (1992). The mesolimbic dopaminergic system is implicated in the reinforcing effects of nicotine. Psychopharmacology (Berl) 107: 285-289.

David SP, Munafo MR, Johansen-Berg H, Mackillop J, Sweet LH, Cohen RA et al (2007). Effects of acute nicotine abstinence on cue-elicited ventral striatum/nucleus accumbens activation in female cigarette smokers: a functional magnetic resonance imaging study. Brain Imaging Behav 1: 43-57.

Day JJ, Roitman MF, Wightman RM, Carelli RM (2007). Associative learning mediates dynamic shifts in dopamine signaling in the nucleus accumbens. Nat Neurosci 10: 1020-1028.

Di Chiara G, Bassareo V, Fenu S, De Luca MA, Spina L, Cadoni C et al (2004). Dopamine and drug addiction: the nucleus accumbens shell connection. Neuropharmacology 47(Suppl 1): 227-241.

Di Chiara G, Imperato A (1988). Drugs abused by humans preferentially increase synaptic dopamine concentrations in the mesolimbic system of freely moving rats. Proc Natl Acad Sci USA 85: 5274-5278.

Due DL, Huettel SA, Hall WG, Rubin DC (2002). Activation in mesolimbic and visuospatial neural circuits elicited by smoking cues: evidence from functional magnetic resonance imaging. $\mathrm{Am}$ J Psychiatry 159: 954-960.

Franklin TR, Wang Z, Wang J, Sciortino N, Harper D, Li Y et al (2007). Limbic activation to cigarette smoking cues independent of nicotine withdrawal: a perfusion fMRI study. Neuropsychopharmacology 32: 2301-2309.

Gomperts BD, Tatham PER, Kramer IM (2002). Signal Transduction. Academic Press: San Diego, California.

Josselyn SA, Nguyen PV (2005). CREB, synapses and memory disorders: past progress and future challenges. Curr Drug Targets CNS Neurol Disord 4: 481-497.

Kenney JW, Gould TJ (2008). Modulation of hippocampusdependent learning and synaptic plasticity by nicotine. Mol Neurobiol 38: 101-121.
Kim JJ, Fanselow MS (1992). Modality-specific retrograde amnesia of fear. Science 256: 675-677.

Lai S, Lai H, Page JB, McCoy CB (2000). The association between cigarette smoking and drug abuse in the United States. J Addict Dis 19: 11-24.

Le Foll B, Goldberg SR (2005). Control of the reinforcing effects of nicotine by associated environmental stimuli in animals and humans. Trends Pharmacol Sci 26: 287-293.

Levin ED, McClernon FJ, Rezvani AH (2006). Nicotinic effects on cognitive function: behavioral characterization, pharmacological specification, and anatomic localization. Psychopharmacology (Berl) 184: 523-539.

Li MD, Lou XY, Chen G, Ma JZ, Elston RC (2008). Gene-gene interactions among CHRNA4, CHRNB2, BDNF, and NTRK2 in nicotine dependence. Biol Psychiatry 64: 951-957.

McClernon FJ, Kozink RV, Rose JE (2008). Individual differences in nicotine dependence, withdrawal symptoms, and sex predict transient fMRI-BOLD responses to smoking cues. Neuropsychopharmacology 33: 2148-2157.

McClung CA, Nestler EJ (2003). Regulation of gene expression and cocaine reward by CREB and DeltaFosB. Nat Neurosci 6: 12081215.

McGeehan AJ, Olive MF (2003). The mGluR5 antagonist MPEP reduces the conditioned rewarding effects of cocaine but not other drugs of abuse. Synapse 47: 240-242.

Miller CA, Marshall JF (2005). Molecular substrates for retrieval and reconsolidation of cocaine-associated contextual memory. Neuron 47: 873-884.

Nestler EJ (2005). Is there a common molecular pathway for addiction? Nat Neurosci 8: 1445-1449.

Pandey SC, Roy A, Xu T, Mittal N (2001). Effects of protracted nicotine exposure and withdrawal on the expression and phosphorylation of the CREB gene transcription factor in rat brain. J Neurochem 77: 943-952.

Phillips RG, LeDoux JE (1992). Differential contribution of amygdala and hippocampus to cued and contextual fear conditioning. Behav Neurosci 106: 274-285.

Picciotto MR, Zoli M, Rimondini R, Lena C, Marubio LM, Pich EM et al (1998). Acetylcholine receptors containing the beta2 subunit are involved in the reinforcing properties of nicotine. Nature 391: 173-177.

Pittenger C, Huang YY, Paletzki RF, Bourtchouladze R, Scanlin H, Vronskaya $S$ et al (2002). Reversible inhibition of CREB/ATF transcription factors in region CA1 of the dorsal hippocampus disrupts hippocampus-dependent spatial memory. Neuron 34: 447-462.

Pluzarev O, Pandey SC (2004). Modulation of CREB expression and phosphorylation in the rat nucleus accumbens during nicotine exposure and withdrawal. J Neurosci Res 77: 884-891.

Robbins TW, Everitt BJ (2002). Limbic-striatal memory systems and drug addiction. Neurobiol Learn Mem 78: 625-636.

Roberts DC, Koob GF, Klonoff P, Fibiger HC (1980). Extinction and recovery of cocaine self-administration following 6-hydroxydopamine lesions of the nucleus accumbens. Pharmacol Biochem Behav 12: 781-787.

Robinson TE, Berridge KC (1993). The neural basis of drug craving: an incentive-sensitization theory of addiction. Brain Res Brain Res Rev 18: 247-291.

Robinson TE, Berridge KC (2003). Addiction. Annu Rev Psychol 54: 25-53.

Schiltz CA, Kelley AE, Landry CF (2005). Contextual cues associated with nicotine administration increase arc mRNA expression in corticolimbic areas of the rat brain. Eur J Neurosci 21: 1703-1711.

Schroeder BE, Binzak JM, Kelley AE (2001). A common profile of prefrontal cortical activation following exposure to nicotine- or chocolate-associated contextual cues. Neuroscience 105 535-545. 
Schultz W, Dayan P, Montague PR (1997). A neural substrate of prediction and reward. Science 275: 1593-1599.

Silva AJ, Kogan JH, Frankland PW, Kida S (1998). CREB and memory. Annu Rev Neurosci 21: 127-148.

Spina L, Fenu S, Longoni R, Rivas E, Di Chiara G (2006). Nicotineconditioned single-trial place preference: selective role of nucleus accumbens shell dopamine D1 receptors in acquisition. Psychopharmacology (Berl) 184: 447-455.

Volkow ND, Wang GJ, Telang F, Fowler JS, Logan J, Childress AR et al (2008). Dopamine increases in striatum do not elicit craving in cocaine abusers unless they are coupled with cocaine cues. Neuroimage 39: 1266-1273.
Walters CL, Blendy JA (2001). Different requirements for cAMP response element binding protein in positive and negative reinforcing properties of drugs of abuse. J Neurosci 21: 9438-9444.

Walters CL, Brown S, Changeux JP, Martin B, Damaj MI (2006). The beta2 but not alpha7 subunit of the nicotinic acetylcholine receptor is required for nicotine-conditioned place preference in mice. Psychopharmacology (Berl) 184: 339-344.

Walters CL, Cleck JN, Kuo YC, Blendy JA (2005). Mu-opioid receptor and CREB activation are required for nicotine reward. Neuron 46: 933-943.

Supplementary Information accompanies the paper on the Neuropsychopharmacology website (http://www.nature.com/npp) 